\title{
Review
}

\section{Leukocyte trafficking in alveoli and airway passages} Claire M Doerschuk

\author{
Case Western Reserve University, Cleveland, Ohio, USA
}

Received: 12 July 2000

Revisions requested: 26 July 2000

Revisions received: 6 September 2000

Accepted: 6 September 2000

Published: 12 October 2000
Respir Res 2000, 1:136-140

(c) Current Science Ltd (Print ISSN 1465-9921; Online ISSN 1465-993X)

\begin{abstract}
Many pulmonary diseases preferentially affect the large airways or the alveoli. Although the mechanisms are often particular to each disease process, site-specific differences in leukocyte trafficking and the regulation of inflammation also occur. Differences in the process of margination, sequestration, adhesion, and migration occur that can be attributed to differences in anatomy, hemodynamics, and the expression of proteins. The large airways are nourished by the bronchial circulation, whereas the pulmonary circulation feeds the distal lung parenchyma. The presence of different cell types in large airways from those in alveoli might contribute to site-specific differences in the molecular regulation of the inflammatory process.
\end{abstract}

Keywords: adhesion molecules, margination, neutrophils, pulmonary inflammation, sequestration

\section{Introduction}

Many of the diseases that affect the lungs involve inflammation. Some diseases, such as asthma, bronchiectasis, and infection with common environmental pathogens, affect primarily the airways, whereas others affect the distal bronchi, bronchioles, and alveoli, including bacterial pneumonia and the acute respiratory distress syndrome. Although many aspects of host defense and the inflammatory response are common to these two sites, there are differences in the mechanisms through which the inflammatory response occurs (Table 1). Many of these differences are related to the particular disease, for example the immune response in asthma compared with innate immunity in bacterial pneumonia, but some of the differences are due to the differences in the circulations and in the structural cell types that form the proximal airways com- pared with the distal air spaces. For example, the airways are fed largely by the bronchial circulation, whereas the distal air spaces are supplied by the pulmonary circulation. In addition, the airway epithelial cells seem to be important in the regulation of the inflammatory response, whereas in the more distal airways the alveolar macrophages and dendritic cells seem important. The purpose of this review is to compare the mechanisms underlying the response of leukocytes to stimuli that affect primarily one of these two lung regions.

\section{Alveoli and distal airways Leukocyte margination}

The distal bronchioles and alveolar spaces are lined by cuboidal epithelium and type I and II alveolar epithelium, respectively, and are fed by the pulmonary circulation. The

ICAM = intercellular cell-adhesion molecule; $\mathrm{VCAM}=$ vascular cell-adhesion molecule. 
pulmonary capillary bed comprises many short interconnecting segments, and a capillary pathway from an arteriole to a venule contains approximately 40-100 capillary segments (reviewed in $\left[1^{\circ}, 2\right]$ ). In normal lungs, this bed contains a large pool of marginated neutrophils, monocytes, and lymphocytes, which are thought to be important in host defense. The concentration of neutrophils in the capillary blood measures 20-60 times the concentration in the large systemic vessels. The transit time of neutrophils through this capillary bed is longer, measuring on average 26-40 s, compared with the transit times of plasma or red blood cells of $1.5-4.0 \mathrm{~s}\left[1^{\bullet}, 2,3^{* *}, 4^{\bullet *}\right]$. This longer transit time is thought to be due to the time required for neutrophils to deform to an oblong shape to pass through the 40-60\% pulmonary capillary segments that are narrower than spherical neutrophils [5]. Measurements of neutrophil shape showed that neutrophils in arterioles were nearly spherical (shape factor of 1.1) whereas neutrophils in the capillary bed had shape factors of $1.5 \pm 0.3$, suggesting that most neutrophils must deform and elongate to travel through the capillary bed $[5,6]$. These studies indicate that the ability to deform is critical in the traffic of neutrophils through normal pulmonary capillaries.

\section{Leukocyte sequestration}

During the acute inflammatory response within regions of the distal lung tissue, neutrophils sequester within the capillary bed. Studies examining the role of known adhesion molecules, including L-selectin, P-selectin, and CD11/CD18, have demonstrated no role for these molecules in the initial stages of sequestration, when neutrophils are stopping and accumulating $[7,8]$. Once neutrophils have stopped, then L-selectin and CD11/CD18 are required to keep the sequestered neutrophils within the capillaries, at least in response to some stimuli.

In many organs fed by the systemic vasculature, neutrophil emigration occurs through the post-capillary venules. Hemodynamic patterns and selectin-mediated rolling of leukocytes are the major mechanisms through which leukocytes sequester at these sites. However, because rolling does not occur within the pulmonary capillary bed and adhesion molecules seem not to be required [6-8], we and others developed alternative hypotheses that focused on the role of the mechanical properties of neutrophils and other leukocytes, particularly on their ability to deform. Observations from many laboratories demonstrated that inflammatory stimuli, particularly those which bind to the seven transmembrane-spanning $G$ protein linked receptors, induce changes in the cytoskeleton of neutrophils that result in a decreased ability to deform $\left[9^{\bullet}, 10^{\bullet}, 11\right]$. These studies led to the hypothesis that stimulus-induced increases in F-actin at the cell periphery led to a decreased deformability, preventing neutrophils from trafficking through the capillary bed and therefore increasing their sequestration at sites of inflammation. The role of
Table 1

\section{Differences between the alveoli and the airways}

Bronchial compared with pulmonary circulation

Structure of vessels

Expression of adhesion molecules

Size and hemodynamic characteristics of the site of leukocyte emigration

Structural differences in alveolar walls compared with bronchial walls Cell types present

Anatomy and properties of the extracellular matrix

Response of structural cells at each site to inflammatory stimuli Mediators released (cytokines, chemokines, and others)

Adhesion molecules expressed and used

Contribution of biomechanical events

Stimuli and disease processes affecting alveoli compared with airways

these changes in vivo was suggested by observations demonstrating that neutrophils sequestered in the pulmonary capillaries of rabbits after the infusion of complement protein 5 fragments were rounder than neutrophils in the capillaries of control rabbits [9*]. Although the hypothesis that this increased stiffness and decreased ability to deform mediates the initial sequestration of neutrophils within the pulmonary capillary bed at sites of inflammation has been difficult to prove directly, a considerable number of circumstantial data have accumulated that support this concept. Current studies are focused on understanding the mechanisms through which inflammatory mediators induce this rearrangement of the cytoskeleton.

The cytoskeletal changes that mediate the decreased deformability seem transient, and adherence between the neutrophils and the endothelial cells occurs within 5-30 min. The mechanical properties of these adherent and crawling neutrophils become very complex. Yanai et al [12] have demonstrated, with the use of optical tweezers, that granules within the cytoplasm of the leading edge are easier to oscillate than granules in the body or the trailing region of neutrophils. Schmid-Schoenbein et al [13] have suggested that pseudopods have more rigid viscoelastic properties than the remainder of the leukocyte. A full understanding of the biochemical and mechanical events that occur during adhesion and crawling has not yet emerged but is an important topic of investigation; the events might be modulated in part through signaling by ligated adhesion molecules. Neutrophil adhesion to endothelial cells induces changes in both neutrophils and endothelial cells that result in cytoskeletal and other intracellular alterations that are probably required for the subsequent steps in the response of neutrophils. For example, when neutrophils bind to pulmonary microvascular endothelial cells through intercellular cell-adhesion molecule-1 (ICAM-1), ICAM-1-dependent intracellular signaling responses are initiated [14]. This signaling response 
seems to require reactive oxygen species generated by xanthine oxidase, and results in the remodeling of the cytoskeleton through signaling pathways that are only now being explained.

\section{Leukocyte emigration}

Following sequestration and initial adhesion, neutrophils migrate along the capillary endothelium and into the interstitium and alveoli. There are at least two pathways through which this emigration occurs, one that uses the leukocyte adhesion molecule CD11/CD18 and one that does not. Which adhesion pathway is selected appears to depend upon the stimulus (reviewed in [11]). Stimuli that induce CD18-dependent emigration include Escherichia coli, E. coli lipopolysaccharide, Pseudomonas aeruginosa, IgG immune complexes, interleukin-1, and phorbol myristate acetate. These stimuli seem to act by inducing the translocation of nuclear factor- $\mathrm{KB}$, resulting in the production of inflammatory cytokines (including tumor necrosis factor- $\alpha$ ) and ICAM- 1 on the pulmonary capillary endothelial cells $[11,15]$. Stimuli that induce CD11/CD18-independent neutrophil emigration include Streptococcus pneumoniae, group B Streptococcus, Staphylococcus aureus, hydrochloric acid, hyperoxia, and C5a. Although the mechanisms through which the CD11/CD18-independent mechanisms occur are less clearly described, neutrophil emigration occurs in the absence of enhanced ICAM-1 expression on the pulmonary capillary endothelium, and interferon- $\gamma$ might be an important regulator of this response. This pathway of neutrophil emigration seems not to require known adhesion molecules, because numerous studies blocking the function of these adhesion molecules, either singly or in combination with blocking antibodies, small molecules, or genetic depletion, have demonstrated no defect in CD11/CD18-independent neutrophil migration. Current studies are focused on identifying new genes by using differential display and gene microarrays to compare transcripts made during CD18dependent and CD18-independent neutrophil emigration.

Neutrophils migrate into the alveolar spaces through a well-defined pathway. The site of sequestration and migration is, as demonstrated by many investigators, the pulmonary capillary bed rather than the post-capillary venules. Neutrophils migrate between endothelial cells through junctions located between the thick and the thin walls of the capillary loops [16*]. This junctional site overlies discontinuities in the basal laminae and is the site of interactions with interstitial fibroblasts within the alveolo-capillary walls. The neutrophils then crawl into the interstitium, where they are often in contact with the fibroblasts and may be crawling along them [17']. They then crawl to sites where type I cells are adjacent to type II cells and preferentially migrate between these two cell types to enter the alveolar space. The process of migration is accompanied by an increase in neutrophil volume, from approximately
$128 \pm 9 \mu \mathrm{m}^{3}$ to $266 \pm 9 \mu \mathrm{m}^{3}$ [18]. Although this increase in volume seems to be mediated through the sodium/ proton antiport, the function of the volume change is not yet clear.

\section{Airways and the bronchial circulation}

Leukocyte trafficking in response to stimuli within the more proximal bronchi occurs through the bronchial circulation. This circulation consists of a capillary network in the subepithelial region of the bronchi and a deeper system of arterioles, capillaries, and venules. The diameter of these capillaries has not been definitively determined under physiological conditions, but they are likely to be wider than pulmonary capillaries. The bronchial circulation displays many attributes of the systemic circulation in general but has an unusual drainage system. More than $85 \%$ of the systemic blood flow to the bronchial circulation returns to the left ventricle, indicating the presence of anastomoses between the bronchial and the pulmonary circulations [19*0. These anastomoses have been identified at the pre-capillary, capillary, and post-capillary levels within the pulmonary circulation.

It has been difficult to establish whether the bronchial vessels contain a marginated pool of neutrophils or other leukocytes. Baile et al [19*0] demonstrated that the fraction of neutrophils requiring a longer time than red blood cells to pass through the bronchial circulation was lower $(50-60 \%)$ than the percentage in trafficking through the pulmonary circulation (80\%). This smaller margination within the bronchial circulation might be due to differences in the anatomy or the hydrodynamic dispersive forces between these two vascular beds. This study might in fact overestimate margination within the bronchial circulation, because a portion of neutrophils could be delayed within the pulmonary microvasculature while traversing the anastomotic pathways. However, these studies do suggest that margination within the bronchial circulation is less than that observed in the pulmonary circulation.

\section{Aspects of the inflammatory response in airways compared with alveoli}

The expression of adhesion molecules in normal lungs and in response to inflammatory stimuli is different in these two circulations. Normally, the pulmonary arterioles and venules express P-selectin, ICAM-1, and vascular cell adhesion molecule-1 (VCAM-1), but not E-selectin, whereas the pulmonary capillaries express only low levels of ICAM-1 and none of the other adhesion molecules $[15,20]$. During the inflammatory response induced by a variety of mediators (including bacteria), the expression of E-selectin, P-selectin, and ICAM-1 is often increased on pulmonary arterioles and venules. However, in the pulmonary capillaries, where neutrophil migration primarily occurs, only ICAM-1 expression is enhanced, and so far only in response to stimuli that elicit CD18-independent 
neutrophil migration. In contrast, in the bronchial microvasculature, P-selectin is expressed on nearly all vessels, and E-selectin, VCAM-1, and ICAM-1 are expressed on many [20]. Inflammatory stimuli induce the upregulation of these molecules. For example, the bronchial microvasculature of asthmatic lungs showed increased expression of selectins, VCAM-1, and ICAM-1, in comparison with nonasthmatic lungs $[21,22]$.

Another difference in the inflammatory response within the large airways from that in the distal lung parenchyma is in the cells that regulate the response. In alveoli, alveolar macrophages and dendritic cells seem to be important modulators of the inflammatory response. The role of type I epithelial cells seems small, although definitive studies have not been performed. Type II cells might provide an important source of mediators regulating the inflammatory response, particularly in the production of surfactant proteins that modulate the inflammatory response and in the production of ICAM-1 on their surface, whose function is still unclear $[15,23]$. In contrast, the many types of epithelial cell lining the airways seem to regulate the response within the bronchi, as well as the lymphocytes, macrophages, fibroblasts, smooth-muscle cells, and other cells within the interstitium, which is much wider than that within the alveolo-capillary walls. Although some chemokines and other mediators might be uniquely produced at only one site, many of the mediators produced in the distal and proximal lung regions are similar. It remains to be determined whether this difference in structural components is important in the regulation of the inflammatory response.

There are considerably fewer studies examining the function of adhesion molecules in bronchial inflammation. During ozone-induced neutrophil emigration, CD11/ CD18, as well as members of the selectin family, seem important in neutrophil emigration [24]. In patients with asthma and animals with airways hyper-reactivity after sensitization and challenge with ovalbumin, the major cell types are lymphocytes and eosinophils. The adhesion molecules in this animal model that seem to be required for the egress of these cells into the interstitium and air spaces include VLA-4/VCAM-1, CD11/CD18-ICAM-1, P-selectin, and E-selectin $[22,25,26]$. To my knowledge, CD11/CD18 is crucial in the emigration of leukocytes through the bronchial circulation, and no CD18-independent pathways have been described.

\section{Conclusion}

There are striking differences in leukocyte trafficking between the alveoli and the proximal airways of the lungs. These differences appear to have multiple etiologies, including differences in anatomy such as the diameter of the vessels and the cell types that regulate the response, differences in both the expression and the function of adhesion molecules by endothelial cells, and differences in the stimuli which induce the inflammatory response at these sites. Although much has been learned about the regulation of inflammation in the past decade, enormous amounts remain to be uncovered, and our current knowledge clearly touches only the tip of the complexities that underlie host defense and inflammation in the lungs.

\section{Acknowledgements}

I thank James C. Hogg, Joseph P. Mizgerd, and Qin Wang for numerous invaluable discussions over many years, and Virginia Ehrbar for help with preparing the manuscript. I apologize to the many scientists whose work I was unable to cite because of limitations on citation numbers. This work was supported by NIH HL 48160, HL 52466 and HL 33009, and a Clinical Scientist Award in Translational Research from the Burroughs Wellcome Fund.

\section{References}

Articles of particular interest have been highlighted as:

- of special interest

$\bullet \quad$ of outstanding interest

1. Hogg JC: Neutrophil kinetics and lung injury. Physiol Rev 1987, - 67:1249-1295.

This paper provides an outstanding review of the pulmonary circulation and neutrophil kinetics.

2. Hogg JC, Doerschuk CM: Leukocyte traffic through the lung. Annu Rev Physiol 1995, 57:97-114.

3. Lien DC, Wagner WW Jr, Capen L, Haslett C, Hanson WL, Hofmeis- ter SE, Henson PM, Worthen GS: Physiological neutrophil sequestration in the lung: visual evidence for localization in capillaries. $J$ Appl Physiol 1987, 62:1236-1243.

These authors were the first to directly measure the transit times of neutrophils through the capillary bed and to describe the manner in which they traversed the bed.

4. Presson, RGJ, Graham JA, Hanger CC, Godbey PS, Gebb SA, Sidner - RA, Glenny RW, Wagner WW Jr: Distribution of pulmonary capillary red blood cell transit times. J App/ Physiol 1995, 79:382-388.

This article describes the frequency distribution of red blood cell transit times through the pulmonary capillaries by using direct visualization and the application of a refined deconvolution technique.

5. Doerschuk CM, Beyers N, Coxson HO, Wiggs B, Hogg JC: Comparison of neutrophil and capillary diameters and their relation to neutrophil sequestration in the lung. App/ Physiol 1993, 74:30403045 .

6. Gebb SA, Graham JA, Hanger CC, Godbey PS, Capen RL, Doerschuk CM, Wagner WW Jr: Sites of leukocyte sequestration in the pulmonary microcirculation. J Appl Physiol 1995, 79:493-497.

7. Doerschuk CM: The role of CD18-mediated adhesion in neutrophil sequestration induced by infusion of activated plasma in rabbits. Am J Respir Cell Mol Biol 1992, 7:140-148.

8. Kubo H, Doyle NA, Graham L, Bhagwan SD, Quinlan WM, Doerschuk CM: L- and P-selectin and CD11/CD18 in intracapillary neutrophil sequestration in rabbit lungs. Am J Respir Crit Care Med 1999, 159:267-274.

9. Motosugi H, Graham L, Noblitt TW, Doyle NA, Quinlan WM, Li Y,

- Doerschuk CM: Changes in neutrophil actin and shape during sequestration induced by complement fragments in rabbits. $A m \mathrm{~J}$ Pathol 1996, 149:963-973.

This paper provides evidence in vivo supporting the hypothesis that decreased deformability is the basis for pulmonary capillary sequestration of neutrophils induced by inflammatory mediators.

10. Worthen GS, Schwab B III, Elson EL, Downey GP: Mechanics of - $\quad$ stimulated neutrophils: cell stiffening induces retention in capillaries. Science 1989, 245:183-186.

These authors provide the first evidence in vivo that inhibiting the decrease in deformability of neutrophils induced by fMet-Leu-Phe prevents their sequestration in the lungs. 
11. Doerschuk CM, Mizgerd JP, Kubo H, Qin L, Kumasaka T: Adhesion molecules and cellular biomechanical changes in acute lung injury. Giles F. Filley Lecture. Chest 1999, 116:37-43S.

12. Yanai M, Butler JP, Suzuki T, Kanda A, Kurachi M, Tashiro H, Sasaki H: Intracellular elasticity and viscosity in the body, leading, and trailing regions of locomoting neutrophils. Am J Physiol 1999, 277: C432-C440.

13. Schmid-Schonbein GW, Skalak R, Sung KLP, Chien S: Human leukocytes in the active state. In White Blood Cells. Morphology and Rheology as Related to Function. Edited by Bagge U, Born GVR, Gaehtgens P. The Hague: Martinus Nijhoff; 1982:21-31.

14. Wang Q, Doerschuk CM: Neutrophil-induced changes in the biomechanical properties of endothelial cells: the roles of ICAM-1 and reactive oxygen species. J Immuno/ 2000, 164:6487-6494.

15. Burns $A B$, Doerschuk CM: Quantitation of L-selectin and CD18 expression on rabbit neutrophils during CD18-independent and CD18-dependent emigration in the lung. J Immunol 1994, 153: 3177-3188.

16. Walker DC, Behzad AR, Chu F: Neutrophil migration through pre-

- existing holes in the basal laminae of alveolar capillaries and epithelium during streptococcal pneumonia. Microvasc Res 1995, 50:397-416.

This paper, in combination with reference 17, provides the first description of the pathway that neutrophils take in migrating from the capillaries to the alveolar space.

17. Behzad AR, Chu F, Walker DC: Fibroblasts are in a position to

- provide directional information to migrating neutrophils during pneumonia in rabbit lungs. Microvasc Res 1996, 51:303-316.

This paper, in combination with reference 16 , provides the first description of the pathway that neutrophils take in migrating from the capillaries to the alveolar space.

18. Worthen GS, Henson PM, Rosengren S, Downey GP, Hyde DM: Neutrophils increase volume during migration in vivo and in vitro. Am J Respir Cell Mol Biol 1994, 10:1-7.

19. Baile EM, Paré PD, Ernest D, Dodek PM: Distribution of blood flow

-. and neutrophil kinetics in bronchial vasculature of sheep. $J \mathrm{App} /$ Physiol 1997, 82:1466-1471.

These authors of this paper compare the transit of neutrophils through the bronchial and the pulmonary circulations, as well as documenting that the drainage of the bronchial circulation occurs primarily into the left ventricle.

20. Feuerhake F, Füchsl G, Bals R, Welsch U: Expression of inducible cell adhesion molecules in the normal human lung: immunohistochemical study of their distribution in pulmonary blood vessels. Histochem Cell Biol 1998, 110:387-394.

21. Hirata N, Kohrogi H, Iwagoe H, Goto E, Hamato J, Fujii K, Yamaguchi $\mathrm{T}$, Kawano $\mathrm{O}$, Ando $\mathrm{M}$ : Allergen exposure induces the expression of endothelial adhesion molecules in passively sensitized human bronchus: time course and the role of cytokines. Am J Respir Cell Mol Biol 1998, 18:12-20.

22. Schleimer RP, Bochner BS: The role of adhesion molecules in allergic inflammation and their suitability as targets of antiallergic therapy. Clin Exp Allergy 1998, 28 Suppl 3:15-23.

23. Wright JR: Immunomodulatory functions of surfactant. Physio/ Rev 1997, 77:931-962.

24. Hyde DM, Miller LA, McDonald RJ, Stovall MY, Wong V, Pinkerton KE, Wegner CD, Rothlein R, Plopper CG: Neutrophils enhance clearance of necrotic epithelial cells in ozone-induced lung injury in rhesus monkeys. Am J Physiol 1999, 277:L1190-L1198.

25. Wolyniec WW, De Sanctis GT, Nabozny G, Torcellini C, Haynes N, Joetham A, Gelfand EW, Drazen JM, Noonan TC: Reduction of antigen-induced airway hyperreactivity and eosinophilia in ICAM1-deficient mice. Am J Respir Cell Mol Biol 1998, 18:777-785.
26. DeSanctis GT, Wolyniec WW, Green FHY, Qin S, Jiao A, Finn P, Noonan T, Joetham AA, Gelfand E, Doerschuk CM, Drazen JM: Reduction of allergic airway responses in P-selectin deficient mice. J App/ Physiol 1997, 83:681-687.

Author's affiliation: Department of Pediatrics, Case Western Reserve University, Cleveland, Ohio, USA

Correspondence: Claire M. Doerschuk, MD, Rainbow Babies and Children's Hospital, Room 787, 11100 Euclid Avenue, Cleveland, OH 44106, USA. Tel: +1 216844 7353; fax: +1 216844 7642; e-mail: cmd22@po.cwru.edu 